\title{
Carrier trapping effects on photoluminescence decay time in InGaN/GaN quantum wells with nanocluster structures
}

\author{
Yen-Cheng Lu, Cheng-Yen Chen, Hsiang-Chen Wang, and C. C. Yang ${ }^{\text {a) }}$ \\ Graduate Institute of Electro-Optical Engineering, National Taiwan University, 1 Roosevelt Road, \\ Section 4, Taipei, 10617 Taiwan, Republic of China and Department of Electrical Engineering, \\ National Taiwan University, 1 Roosevelt Road, Section 4, Taipei, 10617 Taiwan, Republic of China \\ Yung-Chen Cheng \\ Department of Material Science, National University of Tainan, Tainan 700, Taiwan, Republic of China
}

(Received 31 August 2006; accepted 15 January 2007; published online 20 March 2007)

\begin{abstract}
Carrier dynamics in InGaN/GaN quantum wells (QWs) with compositional fluctuations is studied with time-resolved photoluminescence (PL) experiments and Monte Carlo simulations of exciton hopping and recombination. In particular, the effects of indium-rich nanoclusters in such a QW structure on the photon-energy-dependent PL decay time are investigated. In our experiments, two InGaN/GaN QW samples of different silicon doping conditions are used for demonstrating the two cases of different nanocluster densities. An increasing trend of PL decay time on the high-energy side of the PL spectrum is observed in the sample with high nanocluster density. Such a trend is not observed in another sample with few clusters. This difference is consistent with the simulation results which can help us in identifying the origin of the increasing trend as exciton trapping by the local potential minima in the spectral range of the free-carrier states. (C) 2007 American Institute of Physics. [DOI: 10.1063/1.2711148]
\end{abstract}

\section{INTRODUCTION}

Light-emitting devices based on InGaN/GaN quantumwell (QW) structures have a variety of applications, including full-color display and solid-state lighting. However, the mechanisms of efficient luminescence in such a material structure are still not fully understood yet. It has been widely discussed that the highly efficient light emission is due to the indium segregation in the QWs, which produces potential minima for trapping carriers and hence effective radiative recombination. Theories ${ }^{1,2}$ and various experimental results, including the measurements of high-resolution transmission electron microscopy (HRTEM) ${ }^{3-5}$ cathodoluminescence (CL),${ }^{6,7}$ and optical characterizations, ${ }^{8-13}$ have shown the existence of indium-rich nanoclusters, which lie on the background of milder compositional fluctuations, in $\mathrm{InGaN} / \mathrm{GaN}$ QWs.

The details of the carrier dynamics in such nanoclusters are important for understanding the light emission mechanisms in an InGaN compound. Time-resolved photoluminescence (TRPL) experiments for understanding the carrier trapping effects in $\mathrm{InGaN}$ have been reported. Carrier dynamical behaviors in an $\operatorname{In}_{0.20} \mathrm{Ga}_{0.80} \mathrm{~N} \quad(2.5 \mathrm{~nm}) / \mathrm{In}_{0.05} \mathrm{Ga}_{0.95} \mathrm{~N}$ $(6.0 \mathrm{~nm})$ multiple-quantum-well structure have been investigated. ${ }^{11}$ In such a sample, at $20 \mathrm{~K}$ photoluminescence (PL) emission bands with their peaks located at 2.92 and $3.155 \mathrm{eV}$ were observed. Their TRPL experimental results revealed that the emission around $3.155 \mathrm{eV}$ was due to the ground subband-to-subband transition, while the main PL peak at $2.92 \mathrm{eV}$ originated from the excitons localized at the trap centers, which were possibly attributed to the selfformed indium-rich clusters within the QWs.

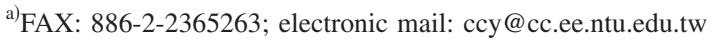

Recently, a nondegenerate femtosecond pump-probe technique has been used for studying the ultrafast carrier dynamics in an InGaN thin film, in which nanoscale indiumrich clusters have been observed with HRTEM and CL. ${ }^{12}$ The carrier capture time of the localized states (the cluster states) from the free-carrier states (the states of the background compound) was calibrated. The initial rise times of the differential transmission of the probe intensity were calibrated to give the time constant of about $300 \mathrm{fs}$ for the degenerate cases over the whole PL spectral range and for the nondegenerate cases, in which both pump and probe wavelengths correspond to the free-carrier states. However, when the carriers were excited in the free-carrier states and probed in the localized states, the rise time increased to the range of 590-715 fs, which represented the carrier capture time of the localized states from the free-carrier states.

Although nanomaterial analysis results, optical characterization data, and theoretical discussions provide quite many evidences of supporting the existences of indium-rich nanoclusters and hence the localized states in $\mathrm{InGaN} / \mathrm{GaN}$ QWs and InGaN thin films, there are still some different opinions about this issue. ${ }^{14,15}$ Cluster-free structures may exist under certain growth conditions. However, indium-rich nanoclusters and significant indium composition fluctuations can exist in other samples under different growth conditions. One more proof based on optical measurement and simulation for the properties of such nanoclusters is useful for clarifying this issue.

In this paper, we demonstrate a feature property of such nanoclusters with the experiment and simulation of the TRPL spectroscopy. From both experimental and simulation results, we observe a feature difference in the photon-energy dependence of PL decay time between two samples of dif- 
ferent cluster densities. The PL decay time variation with photon energy shows an increasing trend on the high-energy side of the time-integrated PL spectrum in a sample with a significant amount of nanoclusters. Such an increasing trend is not observed in another sample with few nanoclusters. The experimental and simulation results are quite consistent with each other.

This paper is organized as follows: In Sec. II, we describe the experimental procedures and results in measuring the TRPL behaviors of the two samples. In Sec. III, we report the simulation techniques for generating the potential structures and exciton hoping process. Then, we present the simulation results in Sec. IV. Here, discussions are also made. Finally, conclusions are drawn in Sec. V.

\section{EXPERIMENTAL PROCEDURES AND RESULTS}

In the experiment, we performed the TRPL measurements of two InGaN/GaN QW samples for comparison. For TRPL measurements, a Hamamatsu streak camera in conjunction with a $25 \mathrm{~cm}$ monochromator was used. The excitation pulses were obtained from the second-harmonic generation of a mode-locked Ti:sapphire laser with a pulse width of $100 \mathrm{fs}$ and a repetition rate of $76 \mathrm{MHz}$. The excitation photon energy, spot size, and power were around $3.3 \mathrm{eV}$, about $2 \mathrm{~mm}$ in diameter, and $3 \mathrm{~mW}$, respectively. The spectral and temporal resolutions of the streak camera system were $1 \mathrm{~nm}$ and $50 \mathrm{ps}$, respectively.

The two InGaN/GaN QW samples of different doping conditions were prepared with metal-organic chemical vapor deposition on (0001) sapphire substrate. Both of them consisted of five QW periods, with $2.5 \mathrm{~nm}$ in well width and $7.5 \mathrm{~nm}$ in barrier width, on top of an undoped $\mathrm{GaN}$ buffer layer of $1.5 \mu \mathrm{m}$ in thickness. The growth temperatures were 1100 and $800^{\circ} \mathrm{C}$ for the $\mathrm{GaN}$ barriers and $\mathrm{InGaN}$ wells, respectively. The average indium content in the wells was estimated to be $10 \%$ in both samples. In sample B, the barriers are silicon doped with the concentration of 5 $\times 10^{18} \mathrm{~cm}^{-3}$. Sample A has the same growth conditions except that there is no doping in any layer. Due to the difference in doping condition, the different strain distributions in the wells led to the distinct nanostructures between the two samples. ${ }^{16}$ Figure 1 shows the results of strain-state analysis (SSA) of samples A and B based on HRTEM. The HRTEM investigations were performed using a Philips Tecnai F30 field emission electron microscope using an accelerating voltage of $300 \mathrm{kV}$ and a probe forming lens of $C_{s}=1.2 \mathrm{~mm}$. Those high-resolution micrographs for SSA were taken with two-electron-beam interference. The current density focused onto the sample was estimated to be lower than $16 \mathrm{~A} / \mathrm{cm}^{2}$. With the two-beam interference data, we could perform the SSA for composition distribution images. ${ }^{17,18}$ In the HRTEM operation, to avoid the possible influence of electron illumination on the recorded results, the images were always taken within 5-10 s after the electron beam was focused onto the target spots. Our experiments showed that the images were not significantly changed within $30 \mathrm{~s}$ of the illumination of focused electron beams. To obtain the SSA images, the [0001] lattice size distributions were first calibrated from the
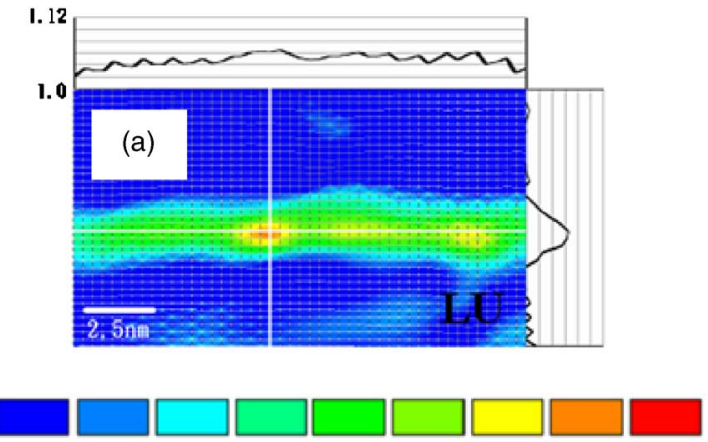

$\begin{array}{lllllllllll}\mathrm{d}_{\mathrm{j}} / \mathrm{d}_{\text {O.N }} & 1.00 & 1.01 & 1.02 & 1.03 & 1.04 & 1.05 & 1.06 & 1.07>1.08\end{array}$

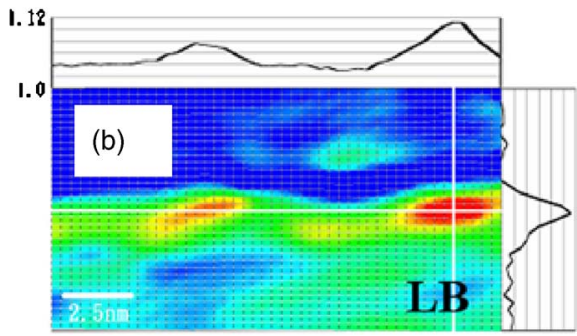

FIG. 1. SSA images of samples A (a) and B (b). $d_{j} / d_{\mathrm{GaN}}=1.04$ corresponds to the indium concentration of about $10 \%$. The indium content variation profiles along the vertical and horizontal line scans are shown.

lattice fringe images. The lattice size distribution is represented by a parameter $d=d_{j} / d_{\mathrm{GaN}}$, defined as the ratio of the local lattice size $\left(d_{j}\right)$ over that of $\mathrm{GaN}\left(d_{\mathrm{GaN}}\right)$. Then, the indium composition distribution can be calibrated based on Vegard's law subject to a control factor. This factor is related to the TEM sample thickness. ${ }^{17,18}$ It is estimated that when $d=1.04$ (1.08), the indium compositions are 10\% (20\%). Therefore, the red spots in Fig. 1 represent the indium-rich nanoclusters. One can see that the nanocluster density in sample B is significantly higher than that in sample A, implying that different silicon doping conditions have led to quite different nanostructures in the InGaN/GaN QWs.

In the TRPL measurements, quite different results between the two samples were also observed. Figure 2 shows the TRPL profiles at $10 \mathrm{~K}$ corresponding to the individual PL spectral peaks of the two samples. One can see that both profiles decay in a single-exponential manner, at least within the first few nanoseconds. In all the measured TRPL profiles, single-exponential decays were observed. Hence, the decay

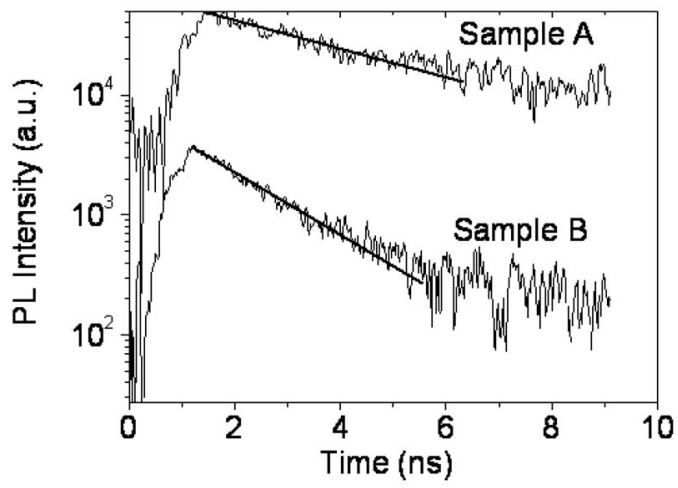

FIG. 2. Experimental TRPL curves at $10 \mathrm{~K}$ at the individual PL spectral peaks of the two samples. 


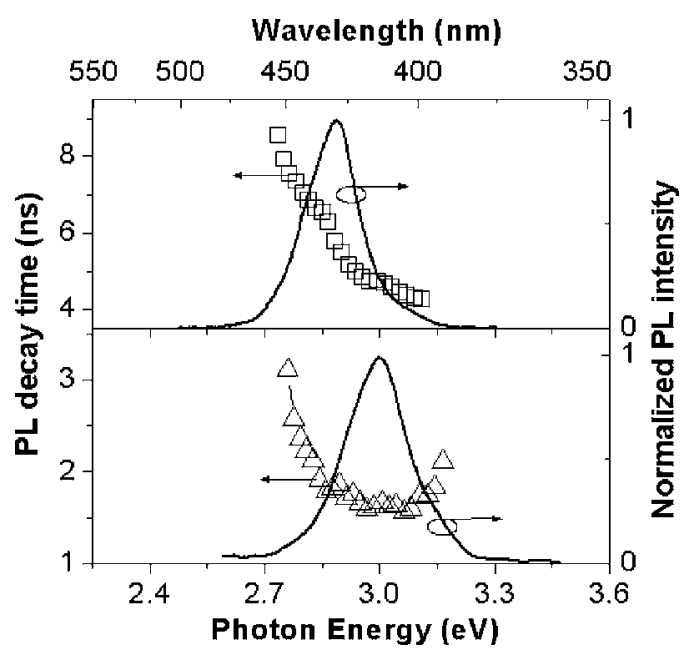

FIG. 3. PL decay time (left ordinate) as a function of photon energy of samples A (a) and B (b). The time-integrated PL spectra (right ordinate) of the two samples are also shown. The small shoulder on the high-energy side of the PL spectrum in part (b) can be clearly seen.

times could be easily calibrated. In Figs. 3(a) and 3(b), we show the photon-energy-dependent PL decay times of samples A and B at $10 \mathrm{~K}$, respectively. The monotonically decreasing trend in sample A and the existence of a minimum in sample B can be clearly seen. In other words, there is an increasing trend of PL decay time on the high-energy side of the time-integrated PL spectrum in sample B. Note that the generally longer decay times $(4-8 \mathrm{~ns})$ in sample A is due to the stronger quantum-confined Stark effect in this sample. ${ }^{19}$

\section{SIMULATION PROCEDURES}

To simulate the conditions of the two samples of high and low cluster densities, we first create two sets of random potential distribution. In the first set, we consider a random spatial variation of potential in which nanocluster does exist. In the second set, on the background of the potential fluctuation similar to the first set, we superimpose the potential variations of randomly distributed nanoclusters with random distributions of size, composition, and position. The potential shape of a cluster is assumed to be in the super-Gaussian form as $\exp \left[-(r / a)^{4}\right]$ based on the cluster shape observed in the HRTEM images. Here, $r$ is the radial coordinate and $a$ can be regarded as the size of a cluster. In the first set, we superimpose periodical potential distributions of different spatial frequencies with random phases in either dimension of a two-dimensional (2D) space. The amplitudes of those spatial frequency components follow a Gaussian spectraldensity function with the peak at zero frequency, the halfmaximum width at $0.5 \mathrm{~nm}^{-1}$, and the peak amplitude at $17.2 \mathrm{meV}$ (corresponding to the standard deviation of potential fluctuation at $110 \mathrm{meV}$ ). In implementing the random potential distribution, the spectral-density function in either the $x$ or $y$ direction is partitioned into 51 components with the step size of $0.04 \mathrm{~nm}^{-1}$ for the aforementioned superposition. A background level of $3.07 \mathrm{eV}$ is added to best fit the experimental data of PL spectrum. Hence, we can obtain a 2D random potential space with a Gaussian-like density-ofstate distribution.

In the second set, we generate super-Gaussian-shaped clusters with random distributions of position, depth, and width on a background level of $3.2 \mathrm{eV}$. The cluster density is $10^{12} \mathrm{~cm}^{-2}{ }^{4}$ Based on the HRTEM images, the random depth and size distributions are assumed to follow Gaussian statistics of $150 \mathrm{meV}$ and $2.5 \mathrm{~nm}$ in mean values, respectively, and $20 \mathrm{meV}$ and $1 \mathrm{~nm}$ in standard deviations, respectively. In Table I, we list all the parameters used for the simulation. Their origins or citations are also shown. The potential realization of the second set is completed by superimposing the cluster distribution onto a background potential fluctuation distribution the same as the first set with the peak amplitude of a Gaussian spectral-density function for the spatial frequency distribution of potential fluctuation at $15 \mathrm{meV}$ for simulating the condition of sample B. In each set, a $2 \mathrm{D}$ potential distribution with $500 \times 500$ pixels (corresponding to $500 \times 500 \mathrm{~nm}^{2}$ ) is realized for carrier relaxation simulations. In Figs. 4(a) and 4(b), we show the potential distribution realizations (only portions of $50 \times 40 \mathrm{~nm}^{2}$ ) of the first and second sets, respectively. Clusters as represented by the dark spots can be clearly seen in Fig. 4(b). Here, we also show a line-scan potential variation in each potential set.

In simulating carrier dynamics at $10 \mathrm{~K}$, we reasonably assume that all carriers are in the form of exciton at $10 \mathrm{~K}$ and the excitons behave similar to single particles in their hopping process between energy states. In the simulation process, the commonly used periodic boundary conditions are employed. ${ }^{20}$ The simulation algorithms of the hopping energy relaxation and the luminescence of exciton recombination follow those suggested by Silver et al. ${ }^{21}$ The hopping transition rate $\nu_{i j}$ from site $i$ to $j$ over a distance $r_{i j}$ is determined by the Miller-Abrahams expression as ${ }^{20,21}$

$$
\nu_{i j}=\nu_{0} \exp \left(-\frac{2 r_{i j}}{\alpha}-\frac{\varepsilon_{j}-\varepsilon_{i}+\left|\varepsilon_{i}-\varepsilon_{j}\right|}{2 k T}\right) .
$$

Here, $\varepsilon_{i}$ and $\varepsilon_{j}$ are the energy levels of the states $i$ and $j$, respectively, $\alpha$ is the decay length of the exciton center-ofmass wave function, and $\nu_{0}$ is the attempt-to-escape frequency. Also, $T$ is the absolute temperature and $k$ is the Boltzmann constant. The used parameters in our simulation include $\nu_{0}=1 \mathrm{THz}, \quad \alpha=0.5 \mathrm{~nm}$, and $T=10 \mathrm{~K}^{20-22}$ The attempt-to-escape frequency $\nu_{0}$ represents the upper limit of the hopping rate. It is chosen to give a reasonable range of hopping rate, which competes with the exciton recombination rate for determining the integrated PL spectral location and shape. If the hopping rate is too high (low) relative to the exciton recombination rate, the PL spectrum will generally be redshifted (blueshifted). Hence, from the experimental data of the PL decay time, we can determine a reasonable range of exciton recombination time. Then, based on the experimental data of the time-integrated PL spectrum, we can obtain a reasonable range of hopping rate. Therefore, the attempt-to-escape frequency can be properly chosen once the exciton decay length is reasonably selected. For the case of 
TABLE I. Parameter values used for simulation and their origins or citations.

\begin{tabular}{|c|c|c|c|}
\hline Parameter & $\begin{array}{l}\text { Case of } \\
\text { sample A }\end{array}$ & $\begin{array}{c}\text { Case of } \\
\text { sample B }\end{array}$ & Origin or citation \\
\hline $\begin{array}{l}\text { Full-width at half-maximum (FWHM) } \\
\text { of the Gaussian spectral-density } \\
\text { function for the spatial frequency } \\
\text { distribution of potential fluctuation }\end{array}$ & $0.5 \mathrm{~nm}^{-1}$ & $0.5 \mathrm{~nm}^{-1}$ & $\begin{array}{l}\text { HRTEM data } \\
\text { and Refs. } 4 \text { and } 16\end{array}$ \\
\hline $\begin{array}{l}\text { Peak amplitude of the Gaussian } \\
\text { spectral-density function for the spatial } \\
\text { frequency distribution of potential } \\
\text { fluctuation }\end{array}$ & $17.2 \mathrm{meV}$ & $15 \mathrm{meV}$ & $\begin{array}{l}\text { HRTEM data } \\
\text { and Refs. } 4 \text { and } 16\end{array}$ \\
\hline Background potential energy level & $3.07 \mathrm{eV}$ & $3.2 \mathrm{eV}$ & PL peak energy data \\
\hline Cluster density & $\cdots$ & $10^{12} \mathrm{~cm}^{-2}$ & $\begin{array}{l}\text { HRTEM data } \\
\text { and Refs. } 4 \text { and } 16\end{array}$ \\
\hline Average of cluster size & $\cdots$ & $2.5 \mathrm{~nm}$ & $\begin{array}{l}\text { HRTEM data } \\
\text { and Refs. } 4 \text { and } 16\end{array}$ \\
\hline $\begin{array}{l}\text { Standard deviation of cluster size } \\
\text { fluctuation }\end{array}$ & $\cdots$ & $1 \mathrm{~nm}$ & $\begin{array}{l}\text { HRTEM data } \\
\text { and Refs. } 4 \text { and } 16\end{array}$ \\
\hline Average of cluster potential depth & $\cdots$ & $150 \mathrm{meV}$ & $\begin{array}{l}\text { PL spectrum } \\
\text { and HRTEM data, } \\
\text { and Refs. } 4 \text { and } 16\end{array}$ \\
\hline $\begin{array}{l}\text { Standard deviation of the fluctuation of } \\
\text { cluster potential depth }\end{array}$ & $\cdots$ & $20 \mathrm{meV}$ & $\begin{array}{l}\text { PL spectrum } \\
\text { and HRTEM data, } \\
\text { and Refs. } 4 \text { and } 16\end{array}$ \\
\hline $\begin{array}{l}\text { Decay length of the exciton } \\
\text { center-of-mass wave function } \alpha\end{array}$ & $0.5 \mathrm{~nm}$ & $0.5 \mathrm{~nm}$ & $\begin{array}{l}\text { HRTEM data } \\
\text { and Refs. 20-22 }\end{array}$ \\
\hline Attempt-to-escape frequency $\nu_{0}$ & $1 \mathrm{THz}$ & $1 \mathrm{THz}$ & $\begin{array}{l}\text { PL and TRPL data, } \\
\text { and Refs. } 20-22\end{array}$ \\
\hline $\begin{array}{l}\text { FWHM of inhomogeneous broadening } \\
\text { spectrum }\end{array}$ & $75 \mathrm{meV}$ & $75 \mathrm{meV}$ & Ref. 26 \\
\hline Exciton recombination time $\tau$ & $6-10 \mathrm{~ns}$ & $\begin{array}{l}1.7-3.7 \mathrm{~ns} \\
\text { (inside cluster) } \\
\quad 6-8 \mathrm{~ns} \\
\text { (outside cluster) }\end{array}$ & $\begin{array}{l}\text { For best fitting } \\
\text { (taking the QCSE into consideration) } \\
\text { and Ref. } 23\end{array}$ \\
\hline
\end{tabular}

sample B, we use the linearly increasing recombination times with decreasing excitation density from 1.7 to $3.7 \mathrm{~ns}$ within the clusters and from 6 to $8 \mathrm{~ns}$ for free-carrier states within the $10 \mathrm{~ns}$ simulation window. There are two reasons for using the shorter recombination time ranges in clusters. The first one is the weaker piezoelectric field and hence higher recombination rate in a cluster. The second reason is the better carrier confinement in a cluster. Here, a cluster domain is defined as a region of potential energy less than $3.05 \mathrm{eV}$. The energy levels above $3.15 \mathrm{eV}$ are defined as the freecarrier states. Between the two energy levels, we use the linear interpolation to obtain the recombination time. The use of increasing recombination times with decreasing exciton density means to take the increasing quantum-confined Stark effect (QCSE) during carrier relaxation (diminishing carrier screening) into account. ${ }^{23}$ In the case of sample A, we also use the linearly increasing recombination time from 6 to $10 \mathrm{~ns}$ because the piezoelectric field is more preserved in this sample. ${ }^{24,25}$ At each position in the $2 \mathrm{D}$ potential distributions, the initial exciton density is assumed to be a constant because we consider a 2D model of only one subband. For each exciton, it may recombine, hop to another site, or not change in a temporal step (1 ps) that is determined by the recombination rate, hopping rate, and a random number. In each temporal step, we record the energy level of the site where an exciton recombines. By collecting those energy levels, we can obtain an emission spectrum. The timeintegrated PL spectrum is then obtained by convolving the spectrum with a Gaussian function of $75 \mathrm{meV}$ in full width at half maximum (FWHM) to take the inhomogeneous broadening effect into account such that we can better simulate the experimental conditions. ${ }^{26}$ 

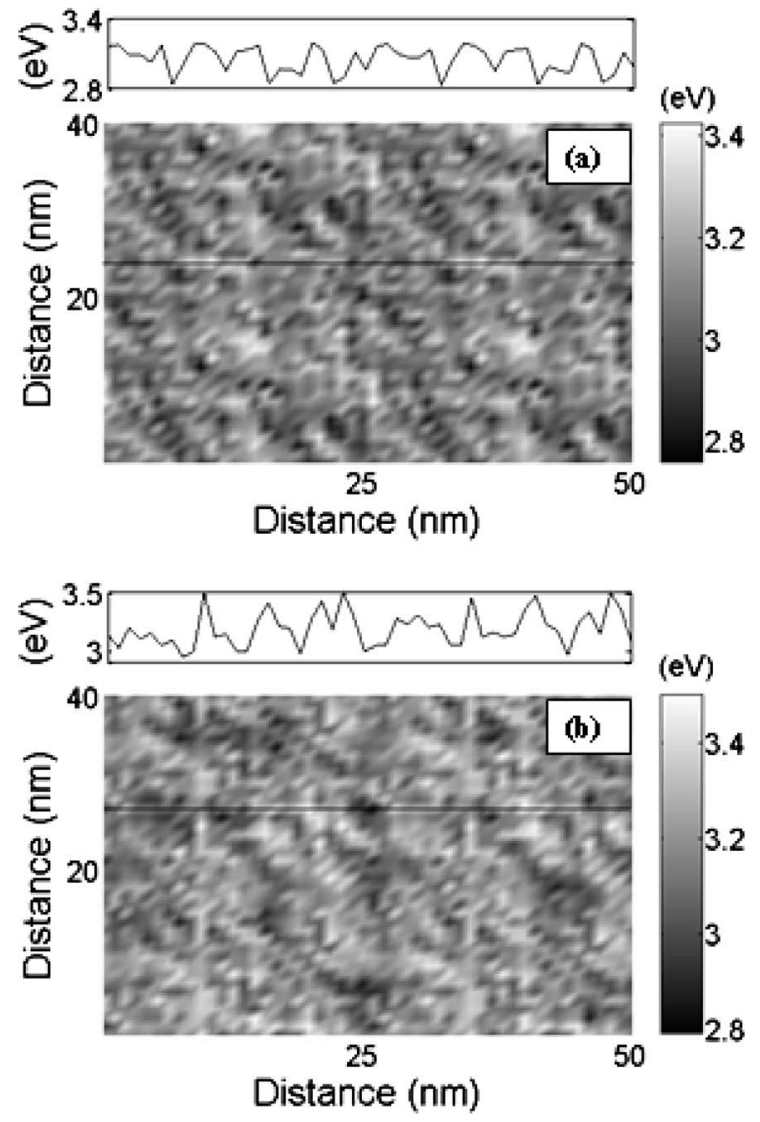

FIG. 4. Simulated potential distributions for the case without randomly distributed nanoclusters (a) and the case with clusters (b). The potential variation profile along a horizontal line scan is shown.

\section{SIMULATION RESULTS AND DISCUSSIONS}

We compare the time-resolved spectra of experimental [part (a)] and simulation [part (b)] results of the case of sample B in Fig. 5. One can see that both sets of spectra are asymmetric tilting toward the high-energy side although the tilt in the simulation result is more significant. Figure 6 shows two TRPL profiles at the individual spectral peaks of the two cases from simulation. The profiles decay single exponentially. The TRPL profiles in Fig. 6 simulate the corresponding experimental results (shown in Fig. 2) quite well. The simulated PL decay times of the two samples are shown in Figs. 7(a) and 7(b) as the thick continuous curves. In these figures, the normalized time-integrated PL spectra (thin continuous curves) and the normalized density-of-state (DOS) distributions (dashed curves) are also plotted. To obtain the DOS distributions, we convolve the energy distributions of the potential realizations with a Gaussian function of $75 \mathrm{meV}$ in FWHM. ${ }^{26}$ In the first case [Fig. 7(a)], corresponding to sample A, the PL decay time decreases monotonically with photon energy that is similar to the experimental data [Fig. 3(a)]. However, in the second case [Fig. 7(b)], corresponding to sample B, the PL decay time variation shows a minimum and then a maximum at the tail on the high-energy side of the PL spectrum. This behavior is again similar to that of the experimental data [Fig. 3(b)]. In experiment, the PL decay time could not be calibrated beyond $3.16 \mathrm{eV}$ because of the low signal-to-noise ratio. Therefore, the decreas-
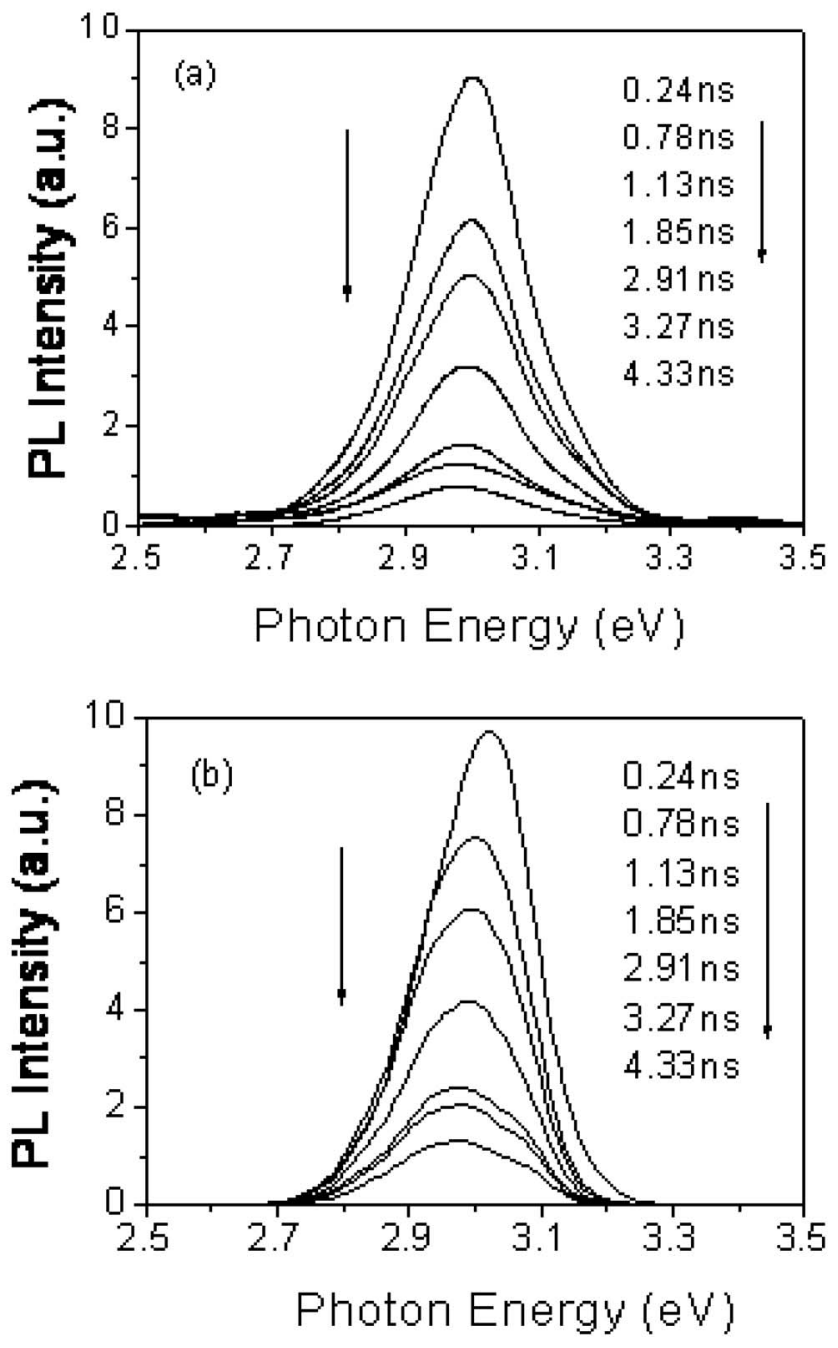

FIG. 5. Time-resolved PL spectra of sample B (a) and the corresponding simulation results (b).

ing trend of PL decay time at the very high-energy end in the experimental data cannot be seen. Nevertheless, the minimum in the thick continuous curve of Fig. 7(b), in contrast to the monotonically decreasing trend of Fig. 7(a), shows the important feature of nanoclusters in the TRPL behavior.

The key reason for observing the minimum of PL decay

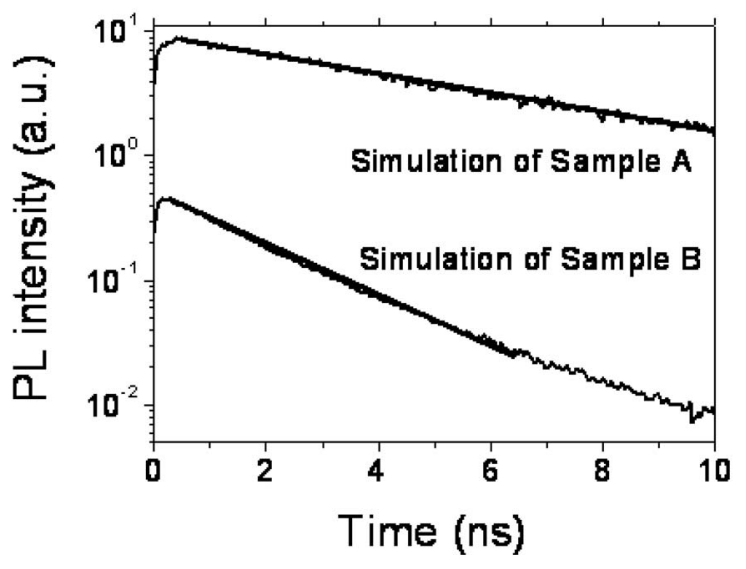

FIG. 6. Simulated TRPL curves at the individual PL spectral peaks of the two cases corresponding to the two samples. 

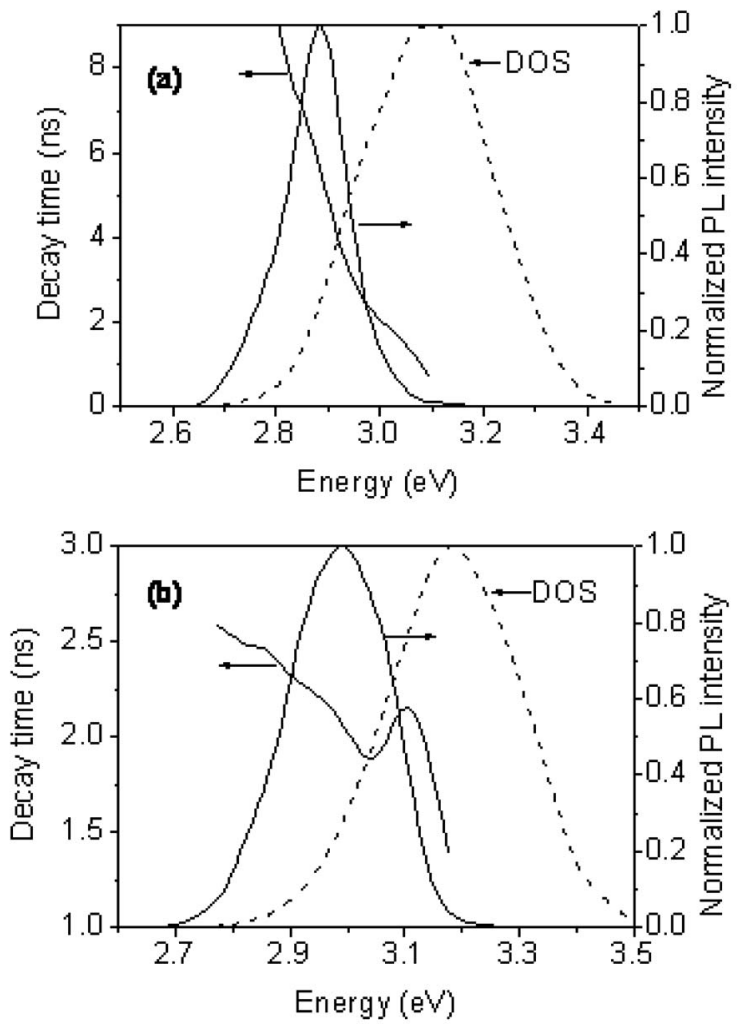

FIG. 7. Simulated PL decay times (thick continuous curves) and normalized time-integrated PL spectra (thin continuous curves) for the case without randomly distributed clusters (a) and the case with clusters (b). The corresponding normalized density-of-state (DOS) distributions are also shown as the dashed curves.

time in varying photon energy in sample B is the existence of the localized states (cluster states). With such states, the major contribution to PL emission originates from the localized states. In this situation, excitons can be trapped by the local minima in the spectral range of the free-carrier states (corresponding to the background composition fluctuations) that results in the elongation of the PL decay time around the low-energy portion of the free-carrier states. This portion corresponds to the high-energy tail of the time-integrated PL spectrum in Fig. 3(b). Therefore, with the clusters, a maximum of the PL decay time at the PL spectral tail can be observed. Note that the PL decay time ranges obtained from the simulations cannot exactly match the experimental data because the theoretical model, such as (1), cannot describe the physical conditions in all details. However, such a minor discrepancy does not affect the key physical features in this research.

Because the processes of exciton hopping and recombination are complicated and random in nature, only the Monte Carlo method can appropriately simulate the real physical scenarios. A simple qualitative or quantitative analysis is insufficient for understanding the detailed mechanisms. In particular, with the simulations of potential realization and exciton hopping, one can gain a picture about the roles of freecarrier states and localized states in contributing to photon emission spectra. Although one can easily conclude that the key reason for observing the minimum of PL decay time in varying photon energy in sample $B$ is the existence of the localized states, the observation of the local maximum of PL decay time here is actually due to the exciton trapping by the local potential minima in the spectral range of the freecarrier states. This conclusion based on our simulation is nontrivial.

\section{CONCLUSIONS}

In summary, carrier dynamics in InGaN/GaN QWs with compositional fluctuations has been studied with the simulation of TRPL behaviors for comparison with experimental data. In particular, the effects of indium-rich nanoclusters in such a QW structure on the photon-energy dependence of the PL decay time were investigated. An increasing trend of PL decay time on the high-energy side of the time-integrated PL spectrum was observed when a high density of nanocluster existed that was not observed in another sample of random potential fluctuations with few clusters. This feature difference is consistent with the experimental result, in which two InGaN/GaN QW samples of different silicon doping conditions were used for demonstrating the two cases of different nanocluster densities. The simulation results demonstrated that the increasing behavior of the PL decay time was due to the exciton trapping by the local potential minima in the spectral range of the free-carrier states. Such a phenomenon does not occur in the case without cluster states.

\section{ACKNOWLEDGMENTS}

This research was supported by National Science Council, The Republic of China, under Grant No. NSC 95-2120M-002-012, and by US Air Force under Contract No. AOARD-06-4052.

${ }^{1}$ I. Ho and G. B. Stringfellow, Appl. Phys. Lett. 69, 2701 (1996).

${ }^{2}$ L. K. Teles, J. Furthmuller, L. M. R. Scolfaro, J. R. Leite, and F. Bechstedt, Phys. Rev. B 62, 2475 (2000)

${ }^{3}$ Y. S. Lin et al., Appl. Phys. Lett. 77, 2988 (2000).

${ }^{4}$ Y. S. Lin et al., Appl. Phys. Lett. 80, 2571 (2002).

${ }^{5}$ M. Rao, D. Kim, and S. Mahajan, Appl. Phys. Lett. 85, 1961 (2004).

${ }^{6}$ S. W. Feng et al., J. Appl. Phys. 95, 5388 (2004).

${ }^{7}$ S. Chichibu, K. Wada, and S. Nakamura, Appl. Phys. Lett. 71, 2346 (1997).

${ }^{8}$ C. K. Choi et al., Phys. Rev. B 63, 195302 (2001).

${ }^{9}$ Y. C. Cheng, E. C. Lin, C. M. Wu, C. C. Yang, and J. R. Yang, Appl. Phys. Lett. 84, 2506 (2004).

${ }^{10}$ H. C. Wang, S. J. Lin, Y. C. Lu, Y. C. Cheng, C. C. Yang, and K. J. Ma, Appl. Phys. Lett. 85, 1371 (2004)

${ }^{11}$ Y. Narukawa, Y. Kawakami, S. Fujita, and S. Fujita, Phys. Rev. B 55, R1938 (1997).

${ }^{12}$ H. C. Wang, Y. C. Lu, C. Y. Chen, and C. C. Yang, Appl. Phys. Lett. 89, 011906 (2006).

${ }^{13}$ A. Kaneta, K. Okamoto, Y. Kawakami, and S. Fujita, Appl. Phys. Lett. 81, 4353 (2002)

${ }^{14}$ T. M. Smeeton, M. J. Kappers, J. S. Barnard, M. E. Vickers, and C. J. Humphreys, Appl. Phys. Lett. 83, 5419 (2003).

${ }^{15}$ P. Ruterana et al., Phys. Status Solidi B 241, 2735 (2004).

${ }^{16}$ M. K. Chen, Y. C. Cheng, J. Y. Chen, C. M. Wu, C. C. Yang, K. J. Ma, J. R. Yang, and A. Rosenauer, J. Cryst. Growth 279, 55 (2005).

${ }^{17}$ D. Gerthsen, B. Neubauer, A. Rosenauer, T. Stephan, H. Kalt, O. Schon, and M. Heuken, Appl. Phys. Lett. 69, 2701 (1996).

${ }^{18}$ A. Rosenauer, U. Fischer, D. Gerthsen, and A. Forster, Appl. Phys. Lett. 71, 3868 (1997).

${ }^{19}$ E. Berkowicz et al., Phys. Rev. B 61, 10994 (2000).

${ }^{20}$ S. D. Baranovskii, R. Eichmann, and P. Thomas, Phys. Rev. B 58, 13081 (1998). 
${ }^{21}$ M. Silver, G. Schonherr, and H. Bassler, Phys. Rev. Lett. 48, 352 (1982).

${ }^{22}$ Y. J. Wang, S. J. Xu, Q. Li, D. G. Zhao, and H. Yang, Appl. Phys. Lett. 88, 041903 (2006).

${ }^{23}$ C. K. Choi et al., Phys. Rev. B 64, 245339 (2001).

${ }^{24}$ M. B. Nardelli, K. Rapcewicz, and J. Bernholc, Appl. Phys. Lett. 71, 3135
(1997).

${ }^{25}$ A. Vinattieri et al., Phys. Status Solidi C 1, 1397 (2004).

${ }^{26}$ K. Kazlauskas, G. Tamulaitis, P. Plbedinskas, A. Zukauskas, M. Springis, C. F. Huang, Y. C. Cheng, and C. C. Yang, Phys. Rev. B 71, 085306 (2005). 